\title{
PROJETO DE UM CORTADOR DE BASE PARA COLHEDORA DE CANA-DE-AÇÚCAR UTILIZANDO OTIMIZAÇÃO MATEMÁTICA ${ }^{1}$
}

\author{
CARLOS E. S. VOLPATO ${ }^{2}$, OSCAR A. BRAUNBECK ${ }^{3}$, CARLOS A. A. de OLIVEIRA ${ }^{4}$, \\ ARISTON P. SANTOS ${ }^{5}$
}

RESUMO: Um cortador de base representado por um mecanismo de quatro barras foi desenvolvido utilizando-se do programa Autocad. Suas partes constituintes foram pré-dimensionadas em função das características operacionais de uma colhedora de cana-de-açúcar em sistema de cana crua e inteira, colhendo uma linha de cana por passada. A força normal de reação do perfil no ponto de contato foi determinada por meio da análise dinâmica, sendo as equações de equilíbrio dinâmico baseadas nas leis de Newton-Euler. O processo de otimização teve como objetivo minimizar a força normal de reação do solo, submetida a restrições de posição, trajetória, comprimento das barras, constante da mola e da força normal. Implementou-se o Algoritmo de Programação Quadrática Seqüencial - SQP do módulo de otimização do programa computacional Matlab. Os resultados mostraram melhora significativa no desempenho de flutuação do mecanismo, representada pela força normal de reação do perfil, a qual foi reduzida de 4.250,33 para 237,13 N. Posteriormente, outras variáveis foram incorporadas ao mecanismo otimizado e um segundo processo de otimização foi implementado.

PALAVRAS-CHAVE: mecanismo de quatro barras, colheita mecânica, projeto de máquinas.

\section{DESING OF A BASE CUTTER FOR SUGAR CANE HAVESTER USING MATHEMATICAL OTIMIZATION}

\begin{abstract}
A base-cutter represented for a mechanism of four bars, was developed using the Autocad program. The normal force of reaction of the profile in the contact point was determined through the dynamic analysis. The equations of dynamic balance were based on the laws of NewtonEuler. The linkage was subject to an optimization technique that considered the peak value of soil reaction force as the objective function to be minimized while the link lengths and the spring constant varied through a specified range. The Algorithm of Sequential Quadratic Programming-SQP was implemented of the program computational Matlab. Results were very encouraging; the maximum value of the normal reaction force was reduced from 4,250.33 to $237.13 \mathrm{~N}$, making the floating process much less disturbing to the soil and the sugarcane rate. Later, others variables had been incorporated the mechanism optimized and new otimization process was implemented.
\end{abstract}

KEYWORDS: four bar linkage, mechanical harvesting, design machine.

\footnotetext{
${ }^{1}$ Projeto Financiado pela FAPEMIG

${ }^{2}$ Prof. Dr., Departamento de Engenharia, UFLA, Lavras - MG, volpato@ufla.br

${ }^{3}$ Prof. Dr., Ph.D., Departamento de Máquinas Agrícolas, FEAGRI/UNICAMP, Campinas - SP, oscar@agr.unicamp.br

${ }^{4}$ Prof. Dr., FESURV, Rio Verde - GO, carlos_aao@yahoo.com.br

${ }^{5}$ Pós-Graduando de Mestrado, DEG/UFLA, aristonps@bol.com.br

Recebido pelo Conselho Editorial em: 8-3-2004

Aprovado pelo Conselho Editorial em: 11-8-2005
} 


\section{INTRODUÇÃO}

Nas colhedoras de cana-de-açúcar e de forragens, o sistema de corte não acompanha o perfil do solo e, dessa forma, não leva em consideração seus desníveis e obstáculos naturais como os sulcos e camalhões. A altura de corte é regulada pelo operador o que acarreta prejuízos financeiros e ambientais pelo corte e carregamento de solo durante a colheita (abaixo do ponto) e pela matéria-prima deixada no campo (corte acima do ponto). Segundo BRAUNBECK (1999), tal situação se resolve por meio do emprego de ferramentas tecnológicas que visam a soluções alternativas e mais arrojadas como modelagem virtual combinada com técnicas de otimização de sistemas mecânicos.

Atualmente, as principais técnicas de otimização de mecanismos tratam a síntese como problema de programação não-linear, em que as soluções ótimas são obtidas por meio da minimização de uma função-objetivo, que é definida como o erro entre as curvas desejada e gerada por um ponto do acoplador. Em termos gerais, pode-se dizer que o problema da otimização consiste em um problema de programação matemática, que pode ser formulado da seguinte maneira: achar o valor das variáveis $\mathrm{X}=\left(\mathrm{x}_{1}, \mathrm{x}_{2}, \ldots \ldots, \mathrm{x}_{\mathrm{n}}\right)$, as quais minimizam ou maximizam, dependendo do que se deseja, determinada função das variáveis mencionadas $\mathrm{Q}=\left(\mathrm{x}_{1}, \mathrm{x}_{2}, \ldots \ldots, \mathrm{x}_{\mathrm{n}}\right)$ denominada função-objetiva. Para tal fim, as variáveis e as funções que as implicam devem estar submetidas às restrições e vínculos do tipo $a_{i} \geq x_{i} \geq$ $b_{i}(i=1,2,3, \ldots \ldots ., n)$. A solução desse problema conduz, então, a extremar a função-objetivo por meio de adequada manipulação das variáveis denominadas variáveis de controle. Existem dois tipos de programação matemática para abordar a solução do problema de otimização: quando a função Q e todas as variáveis dependem linearmente das variáveis de controle $\mathrm{X}$, tem-se um problema de programação linear; se pelo menos uma variável depende não-linearmente das variáveis de controle $\mathrm{X}$, então tem-se um caso de programação não-linear.

Segundo ROOT \& RAGSDELL (1976), a primeira verdadeira otimização de mecanismos é atribuída a Chebychev. Desde os tempos de Chebychev, até os dias de hoje, vários pesquisadores deram sua contribuição no campo da síntese e otimização de mecanismos. Técnicas mais eficientes de otimização para síntese de mecanismos incluem o método de precisão de KRAMER \& SANDOR (1975), a técnica de otimização heurística de DATSERIS \& FREUDENSTEIN (1979), o método do gradiente reduzido generalizado "GRG" de GABRIELE \& RAGSDELL (1989) e a técnica alternativa do método do gradiente reduzido generalizado "GRG" empregando programação quadrática seqüencial.

Mais recentemente, a base para muitos algoritmos de programação não-linear é a solução das equações de Kuhn-Tucker (KT), tentando conseguir o cálculo direto dos multiplicadores de Lagrange. Os métodos Quase-Newton garantem convergência superlinear ao acumularem informações de segunda ordem com relação às equações KT, utilizando, para isso, procedimento de atualização QuaseNewton. Esses métodos são usualmente chamados de métodos de Programação Quadrática Seqüencial (SQP), visto que um subproblema de Programação Quadrática é resolvido em cada iteração principal (POUCAR CASAS, 1998).

Segundo VOLPATO (2001), o método SQP é amplamente difundido na resolução de problemas que envolvem programação não-linear. Inúmeras aplicações desse método em processos de otimização, principalmente para o caso de estruturas podem ser registradas. Segundo ZILLOBER \& ZOTEMANTEL (1994), citados por POUCAR CASAS (1998), os métodos SQP são os algoritmos de uso geral padrão mais usados para resolver problemas de otimização não-linear que cumpram os seguintes pré-requisitos: o problema não seja excessivamente grande; as funções e gradientes possam ser avaliados com alta precisão, e o problema seja bem comportado. O desempenho dos métodos SQP, com relação a outros métodos, é melhor em termos de eficiência, exatidão e percentual de êxito para grande variedade de tipos de problemas testados. 
MURCIA (1998) desenvolveu trabalho com vistas ao dimensionamento otimizado de mecanismos articulados de quatro barras para o seguimento do perfil do solo em processos de colheita de grãos, no qual usaram o "software" de otimização OPT.3.2, conseguindo reduzir o valor da força vertical no garfo cortador de 24,4 para $10,8 \mathrm{~N}$ para um perfil senoidal de solo com amplitude de $0,04 \mathrm{~m}$ e período $0,36 \mathrm{~s}$, coerente com operações agrícolas executadas com velocidades de 1,11 a $2,22 \mathrm{~m} \mathrm{~s}^{-1}$.

Dessa forma, este trabalho teve como objetivo principal minimizar a força normal de reação do solo, submetida a restrições de posição, trajetória, comprimento das barras, constante da mola e da força normal pela implementação do Algoritmo de Programação Quadrática Seqüencial-SQP disponível no programa computacional Matlab.

\section{MATERIAL E MÉTODOS}

\section{Condições pré-otimização}

O projeto inicial do mecanismo de quatro barras, apresentado na Figura 1, foi executado utilizando-se do programa computacional AutoCad. Suas partes constituintes: barra acionadora $\left(\mathrm{L}_{2}\right)$, barra acopladora $\left(\mathrm{L}_{3}\right)$ e barra seguidora $\left(\mathrm{L}_{4}\right)$ - foram pré-dimensionadas em função das características operacionais de uma colhedora de cana-de-açúcar acoplada ao trator agrícola de rodas em sistema de cana crua e inteira, colhendo uma linha de cana por passada.

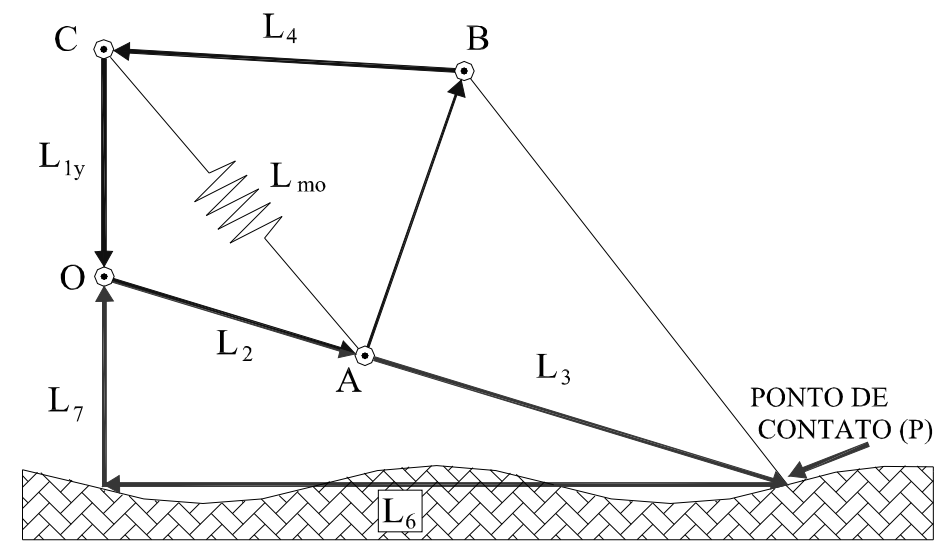

FIGURA 1. Caracterização física do mecanismo de quatro barras.

As propriedades de inércia das peças das barras $\left(\mathrm{L}_{2}\right.$ e $\left.\mathrm{L}_{4}\right)$ e das peças que compõem a barra $\left(\mathrm{L}_{3}\right)$, bem como as dimensões das mesmas foram obtidas pelo próprio AutoCad por meio dos comandos "mass propret" e "dist", conforme Tabela 1. Os valores iniciais da constante da mola $\left(\mathrm{K}_{\mathrm{t}}\right)$ e do seu comprimento inicial $\left(\mathrm{L}_{\mathrm{mo}}\right)$ foram obtidos nas simulações iniciais de forma aleatória (tentativa e erro), porém, com os valores que geravam os resultados mais baixos da força normal de reação. Assim sendo, o valor inicial para a constante da mola foi de $15.000 \mathrm{~N} \mathrm{~m}^{-1}$ e seu comprimento inicial foi de $0,07 \mathrm{~m}$.

TABELA 1. Propriedades de inércia e dimensões das barras de aço estrutural do mecanismo.

\begin{tabular}{cccc}
\hline Barra & Massa $(\mathrm{kg})$ & Momento de Inércia $\left(\mathrm{kg} \mathrm{m}^{-2}\right)$ & Comprimento $(\mathrm{m})$ \\
\hline $\mathrm{L}_{2}$ & 45,1 & 3,8 & 0,501 \\
$\mathrm{~L}_{3}$ & 397,5 & 95,2 & 1,045 \\
$\mathrm{~L}_{4}$ & 11,5 & 4,6 & 0,796 \\
\hline
\end{tabular}


Em seguida, o mecanismo foi, teoricamente, modelado para a obtenção das análises cinemáticas e dinâmicas, pelo método de Newton-Euler, conforme SANTOS (2000). Ao final desse processo, obteve-se um conjunto de equações algébricas cuja resolução se fez necessário o uso de métodos numéricos. Dessa forma, a resolução e as simulações foram feitas no programa computacional científico Matlab, do qual se obteve a força normal de reação do perfil no ponto de contato $\left(\mathrm{F}_{\mathrm{N}}\right)$, adotada como critério de otimização.

\section{Critério de otimização para o mecanismo inicial}

Adotou-se como função-objetivo a força normal de reação do perfil $\left(\mathrm{F}_{\mathrm{N}}\right)$ no ponto de contato com o disco de corte (P), de tal forma que sua magnitude convirja para um valor mínimo e positivo. Essa convergência dar-se-á pelo ajuste das variáveis de projeto à função-objetivo que, neste caso, são constituídas pelo comprimento das barras $\left(\mathrm{L}_{2}, \mathrm{~L}_{3}\right.$ e $\left.\mathrm{L}_{4}\right)$, pelo comprimento inicial da mola $\left(\mathrm{L}_{\mathrm{mo}}\right)$ e pela constante elástica da mola $\left(\mathrm{K}_{\mathrm{t}}\right)$. Também leva-se em consideração as restrições de espaço e trajetória. Três condições iniciais de avaliação foram consideradas nessa etapa para iniciar o processo: velocidade de 2,12 $\mathrm{m} \mathrm{s}^{-1}$; perfil senoidal, e a amplitude do perfil de 0,05 m. Essas foram escolhidas por serem as condições mais severas propostas para serem aplicadas ao mecanismo.

A expressão matemática de entrada para a função-objetivo será escolhida selecionando-se a que mais aproximar os valores da força normal de reação dos objetivos do processo de otimização. As expressões testadas foram:

$(\mathrm{fma})^{2}$ - (fmim $)^{2}$ : minimização do quadrado da amplitude da força;

(fma - fmim): minimização da amplitude da força;

(fma - fmim $)^{2}$ : minimização da amplitude da força ao quadrado;

abs (fma - fmim): minimização da amplitude absoluta da força;

abs (fma)- abs (fmim): minimização absoluta da amplitude da força;

sun $\left(\mathrm{f}_{\mathrm{i}}\right)$ : minimização do somatório das forças ao longo do período, e

sun $\left(\mathrm{f}_{\mathrm{i}}\right)^{2}$ : minimização do somatório das forças ao quadrado ao longo do período.

Os cálculos dos valores da força normal de reação máxima (fma), mínima (fmim), absoluta (abs) e somatório (sun) foram realizados pelas funções específicas existentes no Matlab.

\section{Otimização paramétrica restrita}

Utiliza-se da otimização paramétrica com a finalidade de calcular os valores de um conjunto de parâmetros do projeto definidos pelo vetor $\mathrm{x}=\left\{\mathrm{x}_{1}, \mathrm{x}_{2}, \ldots . ., \mathrm{x}_{\mathrm{n}}\right\}$, que podem, em alguma maneira, ser definidos como pertencentes a um conjunto ótimo. De forma muito simples, o problema pode ser visto como a otimização (maximização ou minimização) de alguma característica do sistema em função do vetor (x). Para o caso em questão, os parâmetros definidos como sendo as variáveis de projeto para otimização foram os comprimentos das barras ( $\left.\mathrm{L}_{1 \mathrm{x}}, \mathrm{L}_{1 \mathrm{y}}, \mathrm{L}_{2}, \mathrm{l}_{3}, \mathrm{~L}_{3} \mathrm{e} \mathrm{L}_{4}\right)$ e a constante da mola $\left(\mathrm{K}_{\mathrm{t}}\right)$, a altura em relação ao perfil $\left(\mathrm{L}_{7}\right)$ e o comprimento inicial da mola $\left(\mathrm{L}_{\mathrm{mo}}\right)$.

O passo inicial para resolver um problema de otimização não-linear com restrições é transformálo em um subproblema mais simples, para em seguida resolvê-lo utilizando como base um processo iterativo. Essa transformação é necessária para a direção de busca durante o processo. Segundo LUENBERGER (1989), um problema qualquer de otimização restrita pode ser expresso como:

Minimizar:

$f(x)$, tal que $x \in R^{N}$

sujeito a: 


$$
\begin{aligned}
& \mathrm{g}_{\mathrm{i}}(\mathrm{x})=0, \quad \mathrm{i}=1, \ldots, \mathrm{m}_{\mathrm{e}} \\
& \mathrm{g}_{\mathrm{i}}(\mathrm{x}) \leq 0, \quad \mathrm{i}=\mathrm{m}_{\mathrm{e}}+1, \ldots, \mathrm{m} ; \\
& \mathrm{x}_{1} \leq \mathrm{x} \leq \mathrm{x}_{\mathrm{u}}
\end{aligned}
$$

em que,

$\mathrm{x}\left(\mathrm{x} \in \mathrm{R}^{\mathrm{n}}\right)$ - vetor de parâmetros do projeto;

$\mathrm{f}\left(\mathrm{f}: \mathrm{R}^{\mathrm{n}} \Rightarrow \mathrm{R}\right)$ - função-objetivo, e

$\mathrm{g}\left(\mathrm{g}: \mathrm{R}^{\mathrm{n}} \Rightarrow \mathrm{R}^{\mathrm{m}}\right)$ - vetor das restrições de igualdade e desigualdade.

Observa-se que um problema de maximização equivale-se a minimizar - $\mathrm{f}(\mathrm{x})$.

O dimensionamento ótimo de mecanismos por meio do processo de otimização contempla restrições de espaço e trajetória, bem como restrições relativas a própria função-objetivo. As restrições usadas neste trabalho foram separadas em dois grupos, conforme suas grandezas adimensionais. Dessa forma, as restrições foram normatizadas e adimensionalizadas para uniformizar a magnitude das grandezas. Assim sendo, surgem dois grupos de restrições:

- Restrições de desigualdade

- constante da mola: $\mathrm{Kt} \geq 0, \mathrm{~N} \mathrm{~m}^{-1}$;

- força normal de reação do perfil: $\mathrm{F}_{\mathrm{N}} \geq 0, \mathrm{~N}$;

- trajetória angular: $5,76 \leq \theta_{3} \leq 5,96$, rad, e

- comprimento inicial da mola: $0,1 \leq \mathrm{L}_{\mathrm{mi}} \leq 0,3$, m.

- Restrições de contorno

- Comprimento das barras:

- Limite inferior (vlb): $0,0\left[\begin{array}{llllll}\mathrm{L}_{1 \mathrm{y}} & \mathrm{L}_{2} & \mathrm{l}_{3} & \mathrm{~L}_{3} & \mathrm{~L}_{4}\end{array}\right], \mathrm{m}, \mathrm{e}$

- Limite superior (vub): 2,0 [ [ [ $\left.\begin{array}{lllll}\mathrm{L}_{1 \mathrm{y}} & \mathrm{L}_{2} & \mathrm{l}_{3} & \mathrm{~L}_{3} & \mathrm{~L}_{4}\end{array}\right], \mathrm{m}$.

As restrições de espaço aparecem devido à necessidade que existe do novo dimensionamento ser compatível aos limites de espaço na máquina. As restrições de trajetória aparecem para garantir a trajetória reta do extremo do disco cortador de base durante os estágios de retração e elevação da ponta do disco. As restrições relativas à função-objetivo tem por finalidade garantir o valor positivo da força.

\section{Algoritmo base de implementação}

Os processos e programas de apoio implementados sobre o algoritmo original do método SQP do pacote computacional científico Matlab foram feitos no próprio Matlab. Esses são apresentados na Figura 2 em forma de fluxograma. 


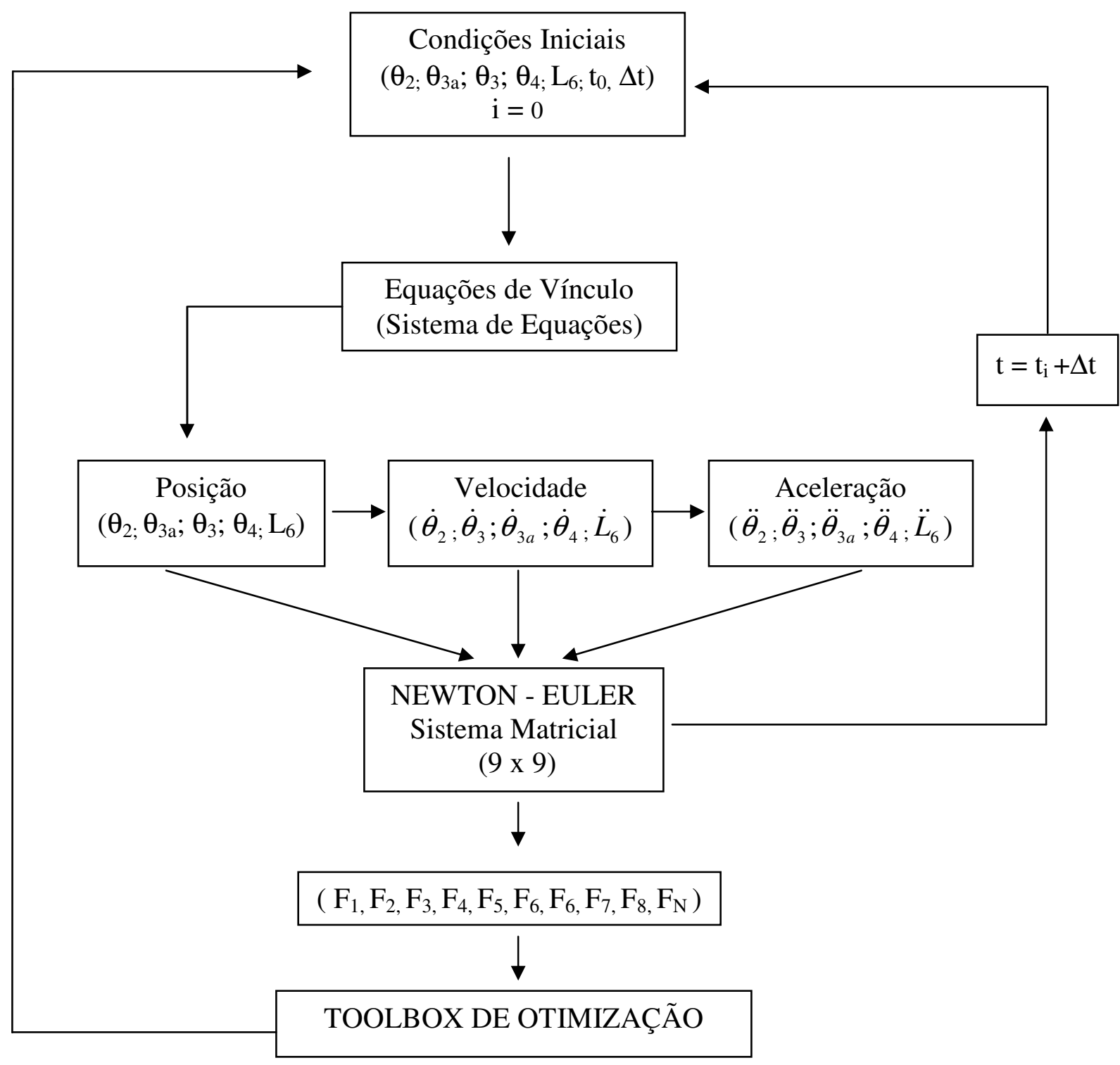

FIGURA 2. Fluxograma do programa de otimização implementado pelo programa computacional científico Matlab.

\section{Condição de pós-otimização}

Após a otimização, as simulações mostraram espaço para refinamento orientado ao aprimoramento do desempenho observado, de onde foram incorporadas novas variáveis ao projeto original, gerando o mecanismo pós-otimizado. As variáveis de projeto escolhidas para essa fase correspondem a componentes periféricos, como a mola e o amortecedor da suspensão, os quais não requerem mudanças estruturais para sua incorporação ao projeto do mecanismo.Verificou-se, pelas simulações iniciais, que existe nível de amortecimento baixo no mecanismo, no valor de $304 \mathrm{~N} \mathrm{~s} \mathrm{~m}^{-1}$, amortecimento esse equivalente a um amortecedor viscoso instalado verticalmente no ponto de contato do disco cortador com o perfil. A constante de amortecimento foi considerada como variável de projeto por se tratar de um parâmetro clássico que afeta significativamente o desempenho dinâmico do mecanismo. Analisou-se, também, a massa da barra $\mathrm{L}_{3}$ como variável de projeto, visando a verificar o 
potencial de ganho existente nessa variável durante o desenvolvimento de um projeto de segunda geração. Para a pós-otimização, em todos os casos, o processo foi submetido às seguintes restrições:

- Valor mínimo da força de reação do perfil $\geq 10 \mathrm{~N}$;

- Constante elástica da mola: $\mathrm{K}_{\mathrm{t}} \geq 0, \mathrm{~N} \mathrm{~m}^{-1}$;

- Comprimento ativo da mola: $\mathrm{L}_{\mathrm{mo}} \geq 0, \mathrm{~m}$;

- Distância de montagem externa da mola: $0 \leq \mathrm{D}_{\mathrm{me}} \leq 8$, m;

- Coeficiente de amortecimento viscoso: $\mathrm{C}_{\mathrm{p}} \geq 0 \mathrm{~N} \mathrm{~s} \mathrm{~m}^{-1}$, e

- Altura de trabalho do cortador: $0,850 \leq \mathrm{L}_{7} \leq 1,350, \mathrm{~m}$.

\section{Parâmetros variados para as simulações após a otimização}

Os parâmetros variados para verificação do desempenho de flutuação após o processo de otimização foram os seguintes: tipo de perfil (senoidal, rampa ascendente e rampa descendente); amplitude do perfil $(0,025$ e $0,050 \mathrm{~m})$, e velocidades de avanço da máquina $(0,68 ; 1,12 ; 1,48 ; 1,82$ e $2,20 \mathrm{~m} \mathrm{~s}^{-1}$ ).

\section{RESULTADOS E DISCUSSÃO}

Após vários ensaios, a expressão matemática que melhor representou a função-objetivo para o processo de otimização foi aquela que realizava a minimização da amplitude da força normal ao quadrado, conforme equação $\left(\mathrm{f}_{\mathrm{ma}}-\mathrm{f}_{\mathrm{mim}}\right)^{2}$. Em geral, as expressões que continham forma quadrática foram as melhores tanto em termos de valores positivos mais próximos de zero quanto em comportamento de convergência. $O$ fato da expressão que leva em consideração a amplitude ter sido a melhor se justifica por essa representar melhor a distribuição dos valores da força ao longo do período de tempo, assim como ocorreu em MURCIA (1998). FRIEDLANDER \& MARTINEZ (1992) afirmam que quando se trabalha com função-objetivo ao quadrado, consegue-se retirar o radical contido na expressão da distância entre dois pontos, facilitando, dessa forma, as resoluções numéricas que envolvem derivações, justificando-se a expressão selecionada. Outras expressões, apesar de convergir para um valor mínimo em alguns casos, mostrou-se ineficiente, como o caso daquelas que usavam a função do valor absoluto (abs). Nesse caso, o programa reconhece como sendo o mínimo entre a seqüência de valores $(-5 ;-2 ; 0 ; 2 ; 3$ e 5$)$ o valor zero.

Após os processos de otimização, os novos mecanismos gerados apresentaram novas formas em função das modificações que aconteceram e ficaram definidos conforme aparecem na Figura 3.
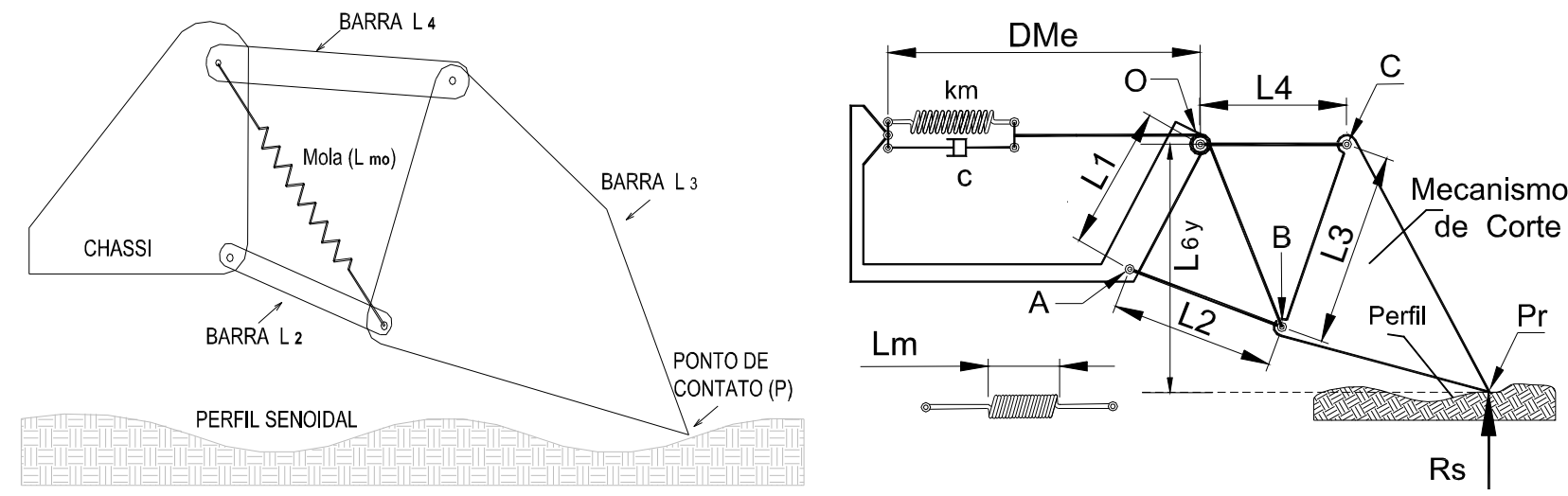

FIGURA 3. Cortador basal sustentado por mecanismo de quatro barras em sua concepção otimizada e pós-otimizada. 
Os novos valores de dimensão obtidos após o processo de otimização para o mecanismo otimizado em comparação com o original encontram-se na Tabela 2.

TABELA 2. Valores das variáveis de projeto entre o mecanismo inicial e otimizado.

\begin{tabular}{cccccccc}
\hline & $\mathrm{L}_{1 \mathrm{Y}}(\mathrm{m})$ & $\mathrm{L}_{2}(\mathrm{~m})$ & $\mathrm{L}_{3}(\mathrm{~m})$ & $\mathrm{L}_{4}(\mathrm{~m})$ & $\mathrm{L}_{7}(\mathrm{~m})$ & $\mathrm{L}_{\mathrm{mo}}(\mathrm{m})$ & $\mathrm{K}_{\mathrm{t}}\left(\mathrm{N} \mathrm{m}^{-1}\right)$ \\
\hline Inicial & 0,600 & 0,716 & 0,796 & 0,947 & 0,544 & 0,700 & 15.000 \\
Otimizado & 0,770 & 0,502 & 1,051 & 0,782 & 0,168 & 0,819 & 103.499 \\
\hline
\end{tabular}

Analisando a Tabela 2, pode-se verificar que, após o processo de otimização, os parâmetros de projetos que mais sofreram alteração em suas dimensões foram a amplitude da articulação inferior, até o ponto de contato com o perfil $\left(\mathrm{L}_{7}\right)$, e constante elástica da mola $\left(\mathrm{K}_{\mathrm{t}}\right)$. A altura influencia na variação de energia acumulada pelo mecanismo, ficando, dessa forma, com valor ótimo o mais próximo do perfil possível, passando de 0,544 para $0,168 \mathrm{~m}$, redução da ordem de $225 \%$. A constante da mola é o parâmetro responsável pela robustez do mecanismo. Observou-se que pequena variação desse parâmetro com valores menores gerava grande variação na força normal de reação do perfil. Daí, o processo ter elevado o valor dessa constante de 15.000 para $103.499 \mathrm{~N} \mathrm{~m}^{-1}$; aumento significativo da ordem de $600 \%$, modificando bastante a forma e atuação desse parâmetro.

Comparando-se o mecanismo inicial e otimizado em termos de variação da força normal de reação, verifica-se que o processo de otimização reduziu de forma significativa o valor dessa força, conforme se pode observar pela Tabela 3 .

TABELA 3. Valores da força normal de reação do perfil em seus pontos máximo, médio, mínimo e de sua amplitude.

\begin{tabular}{ccccc}
\hline Mecanismo & $\mathrm{F}_{\mathrm{N}} \mathrm{m}_{\mathrm{ax}}(\mathrm{N})$ & $\mathrm{F}_{\mathrm{N}} \mathrm{m}_{\mathrm{ed}}(\mathrm{N})$ & $\mathrm{F}_{\mathrm{N}} \mathrm{m}_{\text {in }}(\mathrm{N})$ & Amplitude $(\mathrm{N})$ \\
\hline Inicial & $4.250,33$ & $2.130,22$ & 10,09 & $4.240,24$ \\
Otimizado & 237,13 & 186,81 & 136,65 & 100,48 \\
\hline
\end{tabular}

A redução no valor das forças é bem expressiva, mostrando que o processo de otimização teve êxito em seu objetivo principal, uma vez que os valores obtidos são de magnitude baixa e positiva. A amplitude da força é a resposta mais significativa em termos de análise para o seguimento do perfil. Observa-se redução nesse valor de 4.240,24 para $100,28 \mathrm{~N}$, o que equivale à redução de aproximadamente $4.200 \%$.

TABELA 4. Configurações e condições de otimização dos mecanismos otimizado e pós-otimizado.

\begin{tabular}{ccccccccc}
\hline Mecanismos & $\begin{array}{c}\mathrm{V} \\
\left(\mathrm{m} \mathrm{s}^{-1}\right)\end{array}$ & $\begin{array}{c}\mathrm{A} \\
(\mathrm{m})\end{array}$ & $\begin{array}{c}\mathrm{L}_{\mathrm{mo}} \\
(\mathrm{m})\end{array}$ & $\begin{array}{c}\mathrm{Dme} \\
(\mathrm{m})\end{array}$ & $\begin{array}{c}\mathrm{C}_{\mathrm{p}} \\
\left(\mathrm{N} \mathrm{s} \mathrm{m}^{-1}\right)\end{array}$ & $\begin{array}{c}\mathrm{kt} \\
\left(\mathrm{N} \mathrm{m}^{-1}\right)\end{array}$ & $\begin{array}{c}\mathrm{m}_{3} \\
(\mathrm{~kg})\end{array}$ & $\begin{array}{c}\mathrm{f} \\
(\mathrm{Hz})\end{array}$ \\
\hline Otimizado & 2,12 & 0,050 & 0,819 & 0 & 304 & 103.449 & 397,5 & 1,53 \\
Pós-otimizado & 2,12 & 0,050 & 3,670 & 4,40 & 304 & 12.350 & 162 & 0,20 \\
\hline
\end{tabular}

Após algumas simulações, verificou-se que, em níveis baixos, a constante de amortecimento $\mathrm{C}_{\mathrm{p}}$ tem pouca influência sobre o desempenho de seguimento do mecanismo e o mesmo sofre tendência de não se alterar; conseqüentemente, foi mantido constante o valor de $C_{p}=304\left(\mathrm{~N} \mathrm{~s} \mathrm{~m}^{-1}\right)$. A constante de rigidez da mola $\mathrm{K}_{\mathrm{t}}$ representou a principal variável nesSe novo processo de otimização. Observa-se, na Tabela 4, que o valor de $\mathrm{K}_{\mathrm{t}}$ do mecanismo pós-otimizado é muito inferior ao seu correspondente otimizado, permitindo a implementação de mola com baixa rigidez. Essa condição surgiu devido ao ajuste pelo programa de otimização da distância de montagem externa Dme, que não existia no mecanismo otimizado. A solução constritiva para o ajuste da distância de montagem externa (Dme) 
não representa limitação, uma vez que a mesma pode ser efetuada por meio de cilindro hidráulico de simples ação e pequenas dimensões, aproveitando a infra-estrutura hidráulica existente nas colhedoras ou tratores agrícolas.

A massa da barra $3\left(\mathrm{~m}_{3}\right)$ também apresentou redução expressiva, passando de 397,5 para $192 \mathrm{~kg}$. Esse fator é muito importante sob dois aspectos: redução de gastos na construção e facilidade de operação durante o processo construtivo. Entretanto, essa diminuição provocou significativa redução na freqüência natural do mecanismo, caindo de 1,52 para $0,2 \mathrm{~Hz}$, gerando ângulo de fase de aproximadamente $180^{\circ}$, conforme Figura 4.

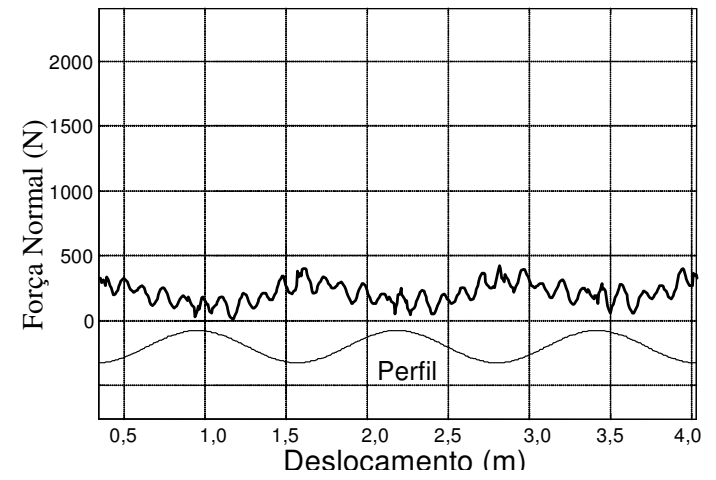

(A)

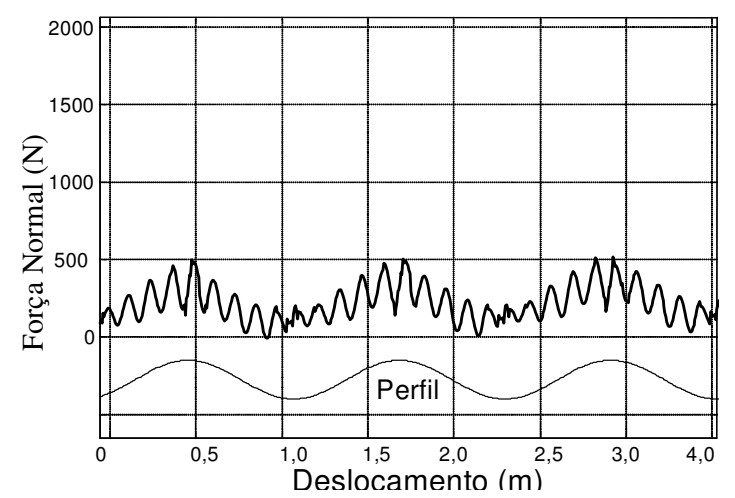

(B)

FIGURA 4. Ângulo de fase de aproximadamente $180^{\circ}$ em função da redução da frequiência natural entre o mecanismo otimizado (A) e pós-otimizado(B).

\section{CONCLUSÕES}

A utilização de processos de otimização matemática em projetos de máquinas agrícolas mostrouse bastante promissora em função dos resultados obtidos. O método SQP obteve soluções com suficiente precisão e rápida convergência. A uniformização da magnitude das grandezas nas restrições melhorou sensivelmente a velocidade de convergência. As variáveis de projeto melhoradas pelo processo de otimização não representam limitações construtivas, uma vez que todas as restrições de espaço e trajetória foram levadas em consideração pelo modelo de otimização. $\mathrm{O}$ mecanismo otimizado obteve bom desempenho de flutuação apenas para condições menos exigentes, como velocidades menores que $1,8 \mathrm{~m} \mathrm{~s}^{-1}$ e amplitude do perfil de $0,025 \mathrm{~m}$. Já para situações mais severas, o mecanismo pós-otimizado conseguiu melhorar esse desempenho devido à introdução da variável Dme.

\section{REFERÊNCIAS}

BRAUNBECK, O.A. Proposta brasileira de colheita mecanizada. In: SEMINÁRIO SOLUÇÕES E NOVIDADES NA MECANIZAÇÃO DA CANA-DE-AÇÚCAR, 1., 1999, Ribeirão Preto. Anais... Ribeirão Preto: IDEA, 1999. p.56-60.

DATSERIS, M.; FREUDENSTEIN, F. Optimum synthesis of mechanisms using heuristics for decomposition and search. Journal of Mechanics Design, Transactions of the ASME, v.101, n.2, p.380-4, 1979.

FRIEDLANDER, A.; MARTINEZ, J.M. New algorithms for maximization of concave functions with box constraints. Rairo - Operations Research, Les Ulis, v.26, n.2, p.209-36,1992.

GABRIELE, G.A.; RAGSDELL, K.M. OPT 3.2. A Nonlinear Programming Code in Fortran Implementing the Generalized Reduced Gradiente Method. User's Manual, 1989. 55 p. 
KRAMER, S.N.; SANDOR, G.N. Selective precision synthesis: a general method of optimization for planar mechanisms. Journal of Engineering for Industry, Transactions of the ASME, v.97, n.2, p.689$701,1975$.

LUENBERGER, D.G. Linear and Nonlinear Programming. $2^{\text {nd }}$ ed. USA: Addison-Wesley Publishing Company. 1989. 491 p.

MURCIA, G.C. Dimensionamento otimizado de mecanismos articulados para o seguimento do perfil do solo em processos de colheita. 1998. 102 f. Tese (Doutorado em Máquinas Agrícolas) Universidade Estadual de Campinas, Faculdade de Engenharia Agrícola, Campinas, 1998.

PAUCAR CASAS, W.J. Concepção ótima de sistemas mecânicos acoplados. Aplicação em problemas elasto-acusticos interiores. 1998. 180 f. Tese (Doutorado em Mecânica dos Sólidos e Projeto Mecânico) - Universidade Estadual de Campinas - Faculdade de Engenharia Mecânica, Campinas, 1998.

ROOT, R.R.; RAGSDELL, K.M. A survey of optimization methods applied to the design of mechanisms. Journal of Mechanics Design, Transactions of the ASME, v.98, n.3, p.1036-41, 1976.

SANTOS, I.F. Dinâmica de sistemas mecânicos: Modelagem, simulação, visualização e verificação. São Paulo: Ed. Makron Books, 2000. 272 p.

VOLPATO, C.E.S. Otimização de um cortador de base para seguimento do perfil de solo em colhedoras de cana-de-açúcar. 2001. 133 f. Tese (Doutorado em Máquinas Agrícolas) - Universidade Estadual de Campinas, Faculdade de Engenharia Agrícola, Campinas, 2001. 